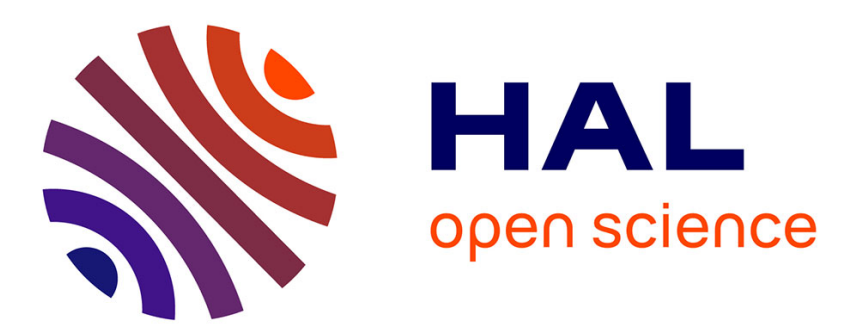

\title{
Trouvailles archéologiques dans la Saône à Anse (Rhône) et aux abords de Chalon
}

\author{
Louis Armand-Calliat
}

\section{To cite this version:}

Louis Armand-Calliat. Trouvailles archéologiques dans la Saône à Anse (Rhône) et aux abords de Chalon. Gallia - Fouilles et monuments archéologiques en France métropolitaine, 1957, 15 (1), pp.128144. 10.3406/galia.1957.1492 . hal-01923724

\section{HAL Id: hal-01923724 \\ https://hal.science/hal-01923724}

Submitted on 3 Mar 2020

HAL is a multi-disciplinary open access archive for the deposit and dissemination of scientific research documents, whether they are published or not. The documents may come from teaching and research institutions in France or abroad, or from public or private research centers.
L'archive ouverte pluridisciplinaire HAL, est destinée au dépôt et à la diffusion de documents scientifiques de niveau recherche, publiés ou non, émanant des établissements d'enseignement et de recherche français ou étrangers, des laboratoires publics ou privés.

\section{(ㅇ)(1) $\$$}

Distributed under a Creative Commons Attribution - NonCommercial - NoDerivatives| 4.0 
des survivances hallstalliennes ont persisté jusqu'il l'époque gallo-romaine dans l'Ouest du départemenl. Fille est plus souvent noire ou grise, ornce de casiers hachurés empreints ${ }^{66}$ ou d'ondulations exéculées au peigne, soit sur le col, soil sur la panse (fig. 8, 16, 25), 28). Ine varianle est l'impression a la molelle de minuscules lriangles, de reclangles, de

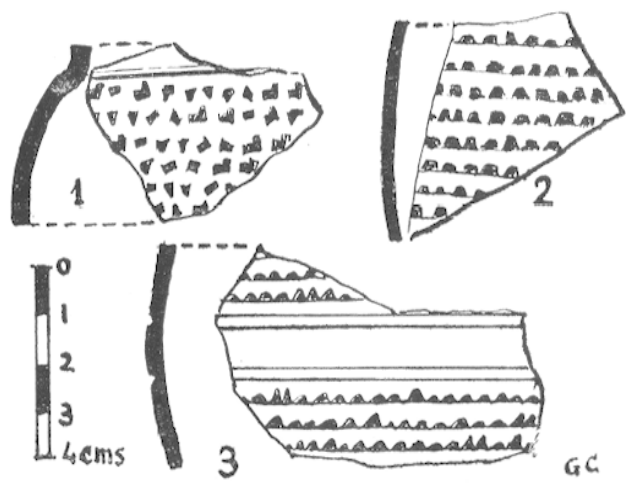

Figr. 9. - Céramique gallo-romaine précoce, grise :nos 2 et 3) et noire $\left(\begin{array}{ll}n^{\circ} & 1\end{array}\right)$.

ronds ou de signes irréguliers en creux (fig. 9, 1,2,3), sur des vases en lerre grise ou noire Iris bien cuile ${ }^{67}$. Quant aux eruches, nous n'en avons trouvé que peu de fragments. Ils sont tous en terre beige

11. 2:21-2:30; La Région du cienlre, 11" 177, 1937, pp. 50)-54; J. Lassus, ciallin, I. 1943, p. 82-91 ; J.-J. H人Tr, ibia., p. 92-124; .V. I. abrotsse, Ciallia, tome VI, 1948, pp. $31-95$.

(66) Presque loujours le potier a passé plusieurs fois sa molette sur les empreintes, puis il a lisse par-dessus et repasse la mollette en appuyant a peine, si bien qu'il est presque impossible par la suite d'identifier la molette qui a servi a faire le travail.

(67) J.-J. HaTr, lissai diune comparaison enlre la ceramique cellique ditulnal sud, elc., dins le Bullelin Mistorique el scienlifique de l'Iurergme, 1.XV, 195., 1. 15\%, fig. 11,1108 . orangé lrès fine, peu cuile, et recouverts d'un engobe blanc très fragile ${ }^{68}$.

Georges ChaRBonneal.

\author{
Trongarlotes archéolocilotis \\ DANS ta SaÓNe a ANSE (Rhònli) \\ hT aUX abordsi me Ghalon
}

1)epuis plusieurs années, les dragages de la saone ont provoupué, sa el li, des découverles archéologiques asse\% nombreuses enlre Lyon of Verdun. Malheureusement la surveillance en est restée plus d'une fois dillicile, le commerce clandestin des anliquilés cherchant trop souvent à s'emparer des objets mis au jour, sans crainle de lausser les indications de provenance et de priver les colleclions publiques des documenls auxqueds elles auraient droil. Grice a la bonne volonté de quelques riverains, il nous sera possible d'examiner ici des trouvailles faites on deux poinls de la mème voie fluviale: à Anse. pres de Villefranche et a Gergy en amont de Chalon. Comme l'analyse du matériel recueilli de part el d'aul.re conduil, en gros, aux mèmes conclusions, il

(6x) J'exprime ma vive seratilude a II. P.-F. Folinsmer, Direcleur de la valle rireonscription archéologrque, qui m'a encourage at aide de ses conseils dans cette tache de longue haleine. Je remereio aussi mes deux didiles collaboraleurs M. E. Brertraxd, pharmacien a Herment, et .I. A. Fourtox, mon gendre, pharmacien a clermont, ainsi que les propriataires des Puy-de-Voingt qui m'ont dommi toute latitude pour fouiller leurs terres.

ces remerciements vont en particulier a .II. Alexis Dians. Félix Pleror, et Raymond Passiblatiole, pour la commune de Voingl, et a .l.M. Fancois RaI\%ET, Raymond L.AporTt.

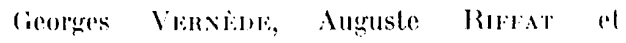
lemri sacastokwsks, pour la rommume de biat. 
a paru intéressant de ne pas séparer l'élude de ces deux sites. Nous avons cru bon égalemenl de leur adjoindre celle d'une anse historiée d'onochoé, draguée en un troisième point, au sud cetle lois de Chaton, mais qui semble peu banale.

\section{ANSE (RHONE)}

Au nord d'Anse, dans la grande courbe que dessine la saine entre ce bourg el Villefranche, M. Rebul, dragueur: a recueilli un cerlain nombre d'objels qu'i la demande de M. Julien (iney, directeur de la XVe circonscription historique il a bien voulu confier pendanl. quelques semaines au Musie de Chalon pour faciliter leur publication'. Le licu précis où ils ont été retirés (fig. 1) se situe sur la rive droile

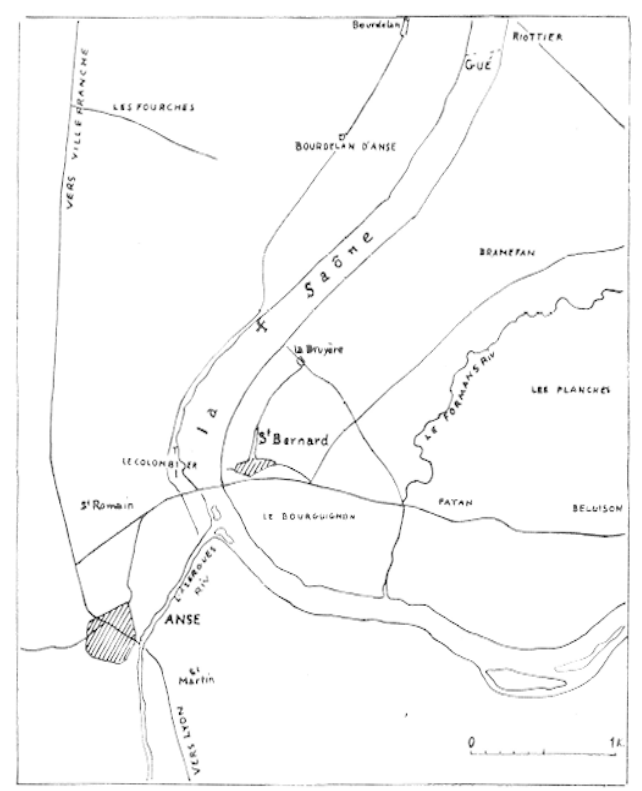

Fig. 1. - Carte de la région d'Anse. La croix indique le point de la saone où ont en lieu les trouvailles.

(1) Ces objets avaient des 1952 attire latlention de s. P. Wuilleumier, alors directeur de la circonscription, qui somhaitait leur étude. Ils ont figuri l'ammie suivanle à l'exposilion de "Ia Salone " organisio a lihalon. Linfin ils onl cle vas en 195.1 a Anse par .H. le J)r linomols. dans le département du Rhòne, en face de Saint-Bernard (Ain), ou plus exactement en face du hameau de La Bruyère, c'est-i-dire à un kilomìtre environ du port des dragueurs d'anse. On peut les classer comme il suil.

Bronze el IIallstall. -- Iluit pièces se rattachent à ces périodes protohistoriques:

1. - Mache à bords droils (fig. $2, n^{\circ} 1$ ). Long. $0 \mathrm{~m}$. 153.3. Le haut est échancré, la partie médiane comme pincée el le tranchanl asse\% élargi. Bronze II de Déchelette. II. N. Savory a donné une carte de la réparlition en France de ce lype qui se renconlre surlout dans le couloir Doubs-Saòne-Rhòne et dans le bassin supéricur de la Garonne; il manque presque complètement dans l'Ouest. Eirnest Chantre en avail déjà signalé un exemplaire a saint-Bernard².

2. - Epée de bronze (fig. $2, n^{0} 2$ a a soie plate avec bords relevés et rivets. Un filet est visible vers la pointe de la lame qui est légirement. pistilliforme. Long. 0) m. 73 . Bronze IV de Décheletle. Barly Hallstalt A de Cowen qui appelle ce type du nom d'Erbenheim³

3. -... Épée de bronze a languelte (fig. $2 n^{0} 3$ ). Long. 0,46. Bronze II-III de Déchelette.

4. Épée de bronze du lype "hongrois" ou "de Liplov" (fig. 2. no 4). Long. 0,53. La garde possede deux rivels et une échancrure demi-circulaire; trois

(2) SAvORY, The sword-bearers, a remterpretation, dans les Proceedings of the Prehisloric sociely, 1948, p. 164 et 169. Е. Cinavrri, Age du Bronze, Lyon, 1875-1876, t. III, p. 16 et Album, pl. IV, 4.

(3) J. 1). Cowsx: The earliest Bronze swords in Brilain and their origin on the continent of Europe, dans les Proresedings citis, lysl, p. 201, 210 el pl. XIV. 


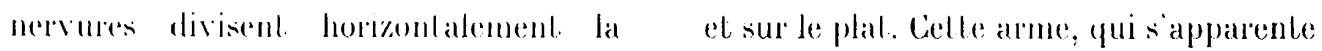
poignée. Iu-dessous de celle-dei, la lame a relles de Beymost el de Villeneuve.
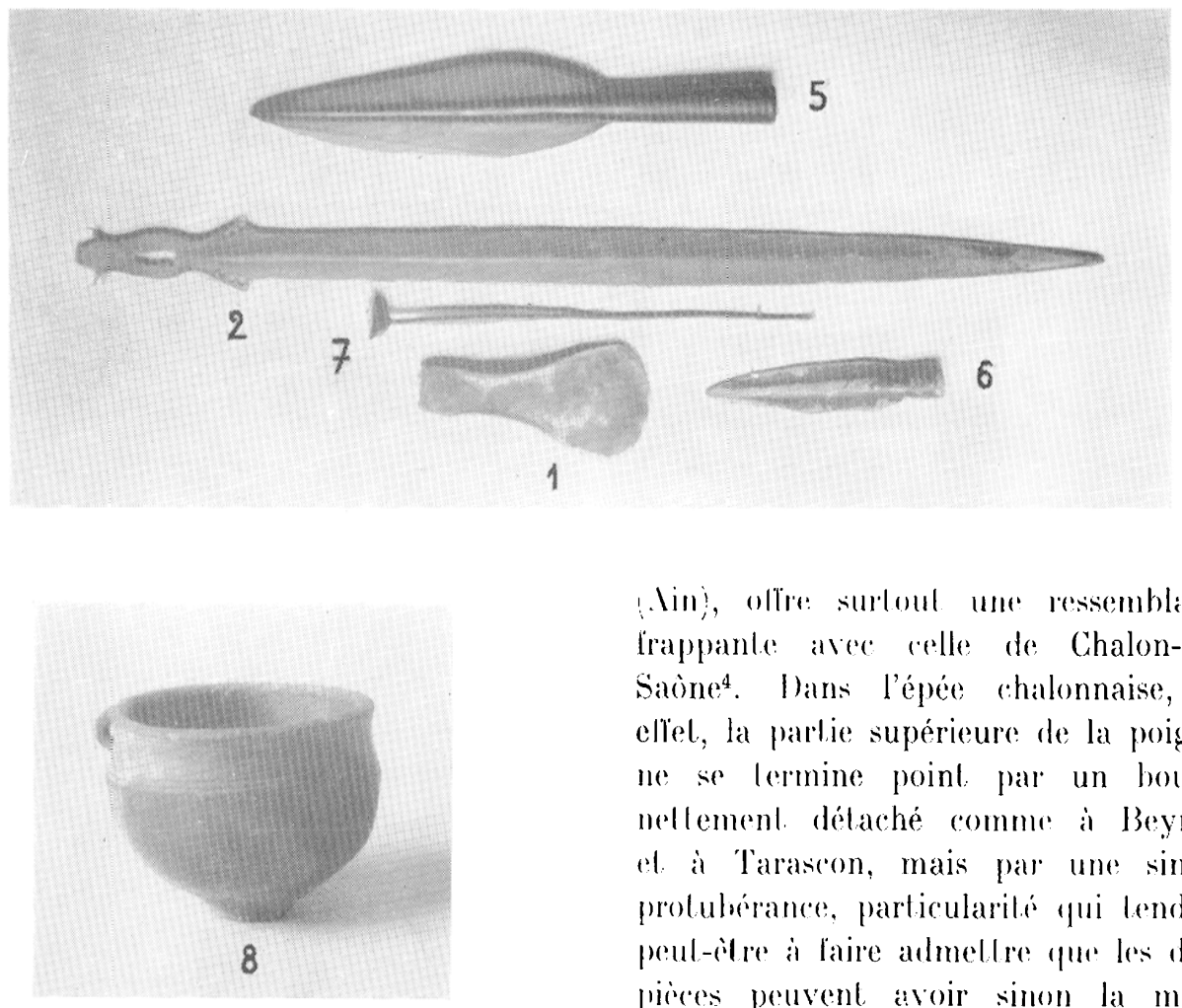

Dinj, ollire sturloul une ressemblance. irappanle aree celle de Chalon-surSaconet. Dans l'épée chalonnaise, en ellel, la parlie supérieure de la poignéc ne se lermine point par un boulon mellement délaché comme a Beynost ef a Taraseon, mais par une simple proluberance, particularitis qui lendrait, peul-alde a laire atmellere que les deux pieces peuvent aroir sinon la mème

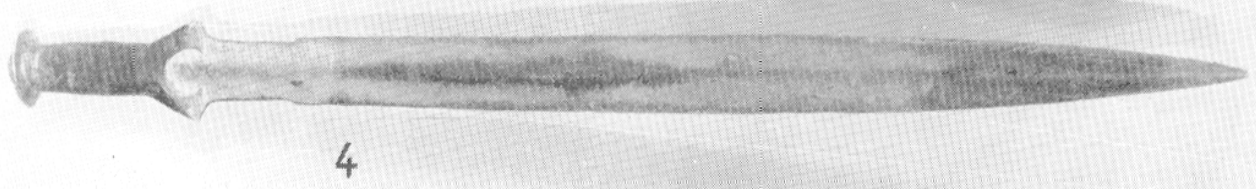

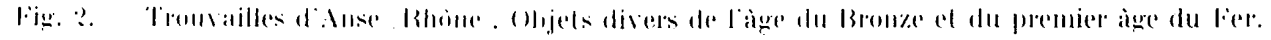

presenter des denleslures lien lines el tres ellaceses, destinese a laciliter la prise par la main du curerriel gui

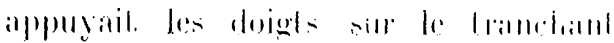

origine, diu moins la mome dale précise d'exérulion. I'une laçon générale en

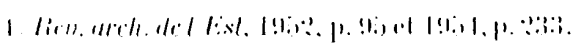


lout cas, ce type nous serail venu de l'Europe cenlrale par le Danube el la suisse. 11. X. Savoryo le rallache $\therefore$ la grande lamille de Möringen dont il a dresse un inventaire des lrouvailles Irangaises oil l'on releve les noms de Belley, Pierre-Chalel, Trévoux el Beynosi, localités silués loules dans le département de l'in. II considere que la date est celle du Hallslall $I$ et $B$, alors que lécheletle rlassail ces glaives au Bronze IV.

5. - Cirande poinle de lance en bronze (fig. 2, no j). Long. 0,37. Deux filels vers le bas de la douille. Celte arme est remarquable par sa conservalion et surtout par ses dimensions supérieures ¿ la moyenne, les lances trouvées en Prance dépassant assez rarement $30 \mathrm{~cm}$. On notera cependant que le Musée de Chalon en possede une plus longue encore qui atteint press de 0,10 el qui provient. de la même rivière; une autre, de la collection chalonnaise également, mesure 0,36 etsort, de la Saòne à Châlenoy-cnBresse.

6. - Seconde pointe de lance en bronze (iig. $\left.2, n^{\circ} 6\right)$. Long 0,17.

7. .. Épingle en bronze à tèle conique dont la tige est finement còtelée à sa partie supérieure (fig. 1, no 7 ). long. 0,32. Bronze III de Jichelette.

8. ... Pelile lasse ou coupe a puiser en lerre noire (fig. 2, no 8 ). Haule de 0.05, pourve d'une ouverture

(5) The sumb-bearers, "reinlerprelalim, dans les Procedings of the Prehisloric Suriely. 19.18, p. 163 et 17.1. Ilme Christiane lassalle a public dans Ie Bulletin des Musées Lyonnais, 1954. p. 26-27, une bonne pholographie de l'exemplaire de vien e a pommeau roneave. de 0,07 , elle est entièrement fabriquée à la main. sans l'aide du tour, et possède une anse línue, modelée avec soin. Le galbe de celte poterie est peu symélrique et les doubles filets qui la cernent doux fois, au-desious et au-desius de la gorge ne sont pas lracés horizonlalement. Comme la lasse en quesilion releve de l'époque de Hallstatt, nous devons nous souvenir que la eommune de sainlBernard, en lace de laquelle ce vase a été dragué, recile un imporlant ensemble de tumulus hallstattiens avec mobilier caractéristique de la civilisation dite des Champs d'urnes, ainsi que l'a chabli le professeur Kimmig'.

La 'lène. Celte période n'a lourni que trois pieces:

1 et 2. - Deux pointes de lance (tig. $\left.3^{\mathrm{a}}\right)$, l'une de 0,35 et l'autre de 0,22 (brisée), en raison de leur nervure centrale tris accentuée et de l'aspect.

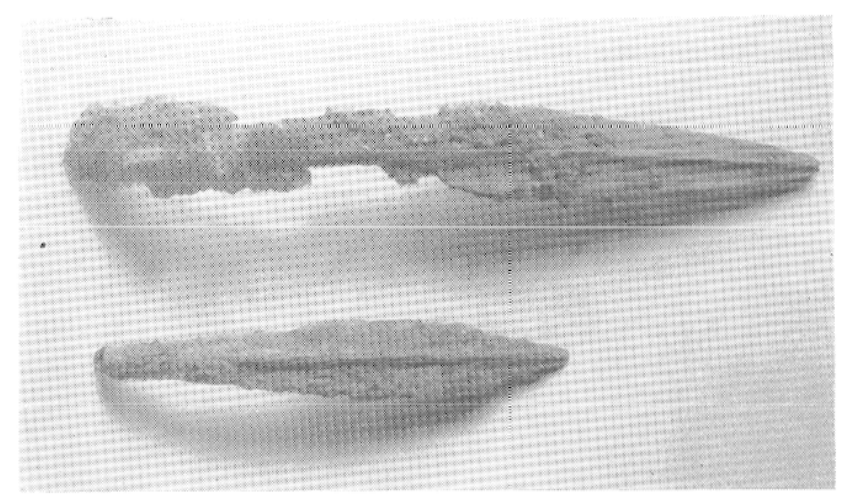

Figr. 3a. Pointes de lance de la Tóne, tromvies a Anse.

du métal, appartiennent au second àge du Fer. Elles sont lres at leintes par la rouille.

i) ReL. arch. ale IEsl. 1952, 1. 167-170.

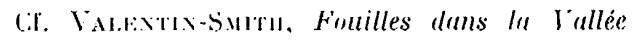
dn Formans (.lin): I.yon, Ixix, pl. I; Claudius SAvori:, le Beaujolais prehistorique, el Décristextes, Manuel, 1 re ed., Agge du Bronze, p. 156. looceupation du platean de Saint-Bernard 
3. $\cdots$ \ la Iroisiome phase de La Tine, nous classerons une assielte à bord rabaltu, parfailement intacle, en terre commune gris-(lair (fig. $3^{\text {b) }}$, avant une hauleur de 0,0\%), el une largeur de 0,49. Ce lype est lrépuent is Bibracle, Gergovie, Marlouxi, ole.

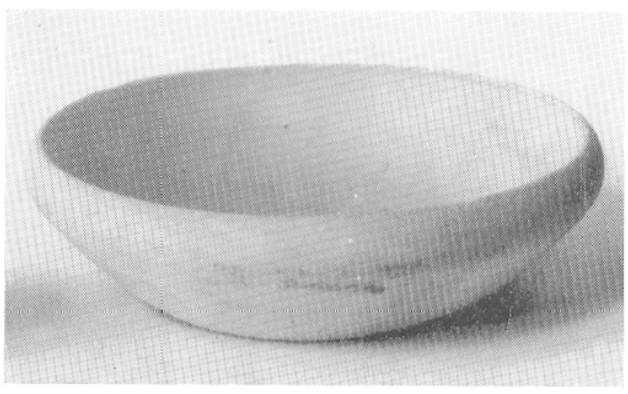

Fig. 3b. - Assielte de l.a Tene III, trouvee a Anse.

Epoque gallo-romaine. Les lemps gallo-romains sont assez abondamment représentés :

1. I.. Ia pieces la plus importanle (fig. 4) est une coupe en bronze coulé, lerminée au lour, alleignant 0.19 de diamelre sur (0,0)is de haut. I l'inlérieur. elle montre un décor incruslé, conslilué par une guirlande d'olivier, faile de deux rameaux donl les feuilles el les tiges sont en argent el les fruils en cuivre rouge. Au centre, et par conséquent loul au creux, se voit une éloile à 6 branches alternativement d'argent el de cuivre rouge. I'extérieur, moins exposé aux regards, n'a pour ornements

va du Neolithique a l'époque de la Tome; en passant far le llallstatt de $A$ a 1$)$ (C:U II a C U IV). - Dans la même région femvirons de Trevoux on connait des tumulus de l'âge du

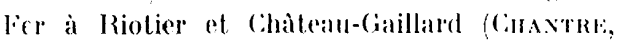
Premier âge du Fer, I.yon-Paris, Is'il, p. 42:.

(7) C.f. HaTr, Apercus sur l'enolulion de la ceramique... dans la Ren. des El. anciennes, t. I.I.

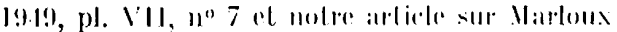
lans Gallia, l!) 14, p. 33. que cinq cerceses concentriques placés au fond et deux aulres qui ourlent. la levre du rebord. I a patine, asse\% épaisse el d'un beau lon verdâtre, a recouvert par malheur une parlie des incrustalions qu'elle rend peu distincles aujourd'hui.

On a déjà remarqué, depuis (irivaud de la Vincelle ${ }^{8}$, que les ouvrages anliques de bronze incrusté d'argenl sont relalivement iréquents enlre Chalon el Iyon. Celle technique décoralive, tris sublile a savante, se relrouve en particulier sur divers usiensiles relires de la saome. dinsi une coupe de la collection Bidaull de Cirésigny, passée dans celle d'liugine schneider, coupe draguée pres de Chalon. offrail une ornementalion idenlique avec les deux branches d'olivier el l'iloile centrale; un plat de la mème collection monlrail deux dauphins aussi incrusles ; un pied cylindrique de staluelle en bronze du Musée de Chalon est. entiorement orné de feruillages d'argent; a un vase de ce Musée est non moins curieusement incrusté d'aregent al do ruive rouge'0. Il nous somvient. entin d'avoir vu chez un garde de navigalion, il y a une lrentaine d'années, un vise lrouvé aussi dans la same pris de Chalon et sur lequel on

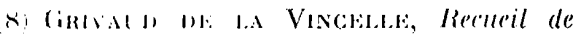
monnmenls anliques, 1. 11, 1817, p. 18:.

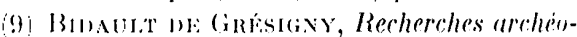
logigues dans la mallée de la Saône, Chalon, 192:1, pl. XXXIV et XXXV. (es deux pieces ont iti trouvies un peu au sud de Chalon. billes daient conservies avant la guerre an Crensot dans lo chatean de la lerrerie.

(10) Nous avons publie ce vase dans le falulogue... du musce de Chalon, 1950, pl. 1V, $11^{\circ} 398$, et le piedestal dans notre Chalonnais galloromain, 19:37, pl. I. I.e même nusce s'est enrich! demierement diun houchom de vase en bromze. presentanl des placages dargent en forme do

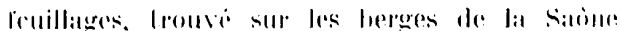
pros du Polit-dacusol, à Chaton mème. 


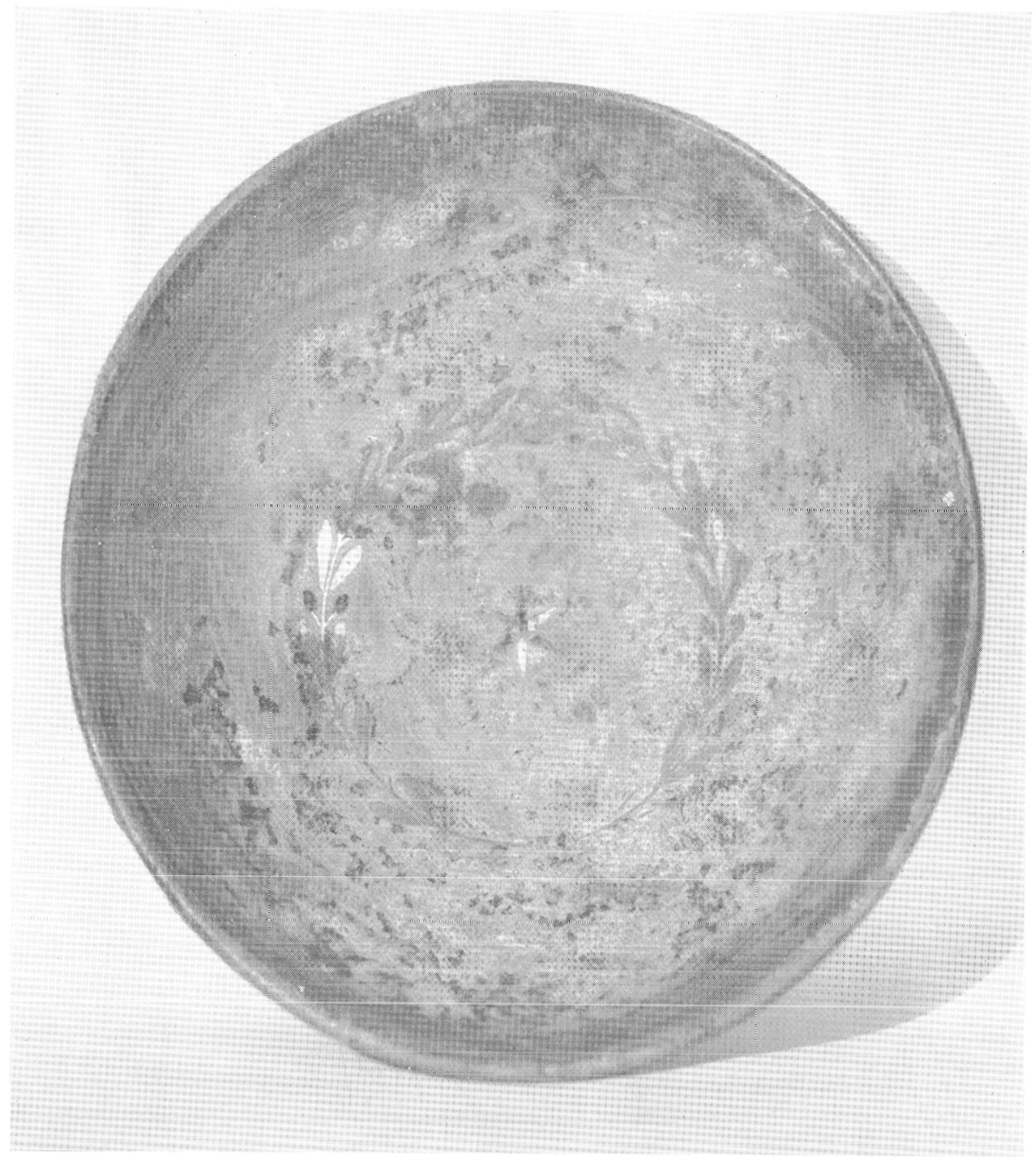

Fig. 4. Coupe en bronze, incrustee dargent et de cuivre rouge, drague a Anse.

remarquait, toul autour, des motifs d'argenl el de cuivere rouge, Iraités avec: ce procédé qui ne semble pas avoir été très souvent employé ailleurs, pour la décoralion des uslensiles, d'une facon aussi systématique, bien qu'on le remarque par exemple sur les anses de trois renorhofis de Cabinel des Médailles, sans provenaner précise connuell, el sur des vases du Musée des Antiquites nationales ${ }^{12}$. lin effet,

(11) Bablidon et BLaxcher, Calalogue des bronzes de la Bibl. nat., n० 1393 (Cabinet de SainteGeneviive, 1394 (Cabinet de l'intendant Fourault, 13966 tsalns origine ancune?

I: S. Risixan. Bronzes figures, t" 106 Neris, lls (Bardonis), 41 (Santenay, cote- 
il s'agil pluloil, dans ces dermiers cas, d'objels d'arl richement diselés où l'incrustalion ne jour: qu'un röle secondaire, alors que, pour les bronzes rhatonnais, elle est unique ol essenlielte.

2. Ln grand vase en brom\%e dont l'arise manque (tig. 5) $)^{\text {il }}$ esl identique a un autre dragué prise de Chaton en

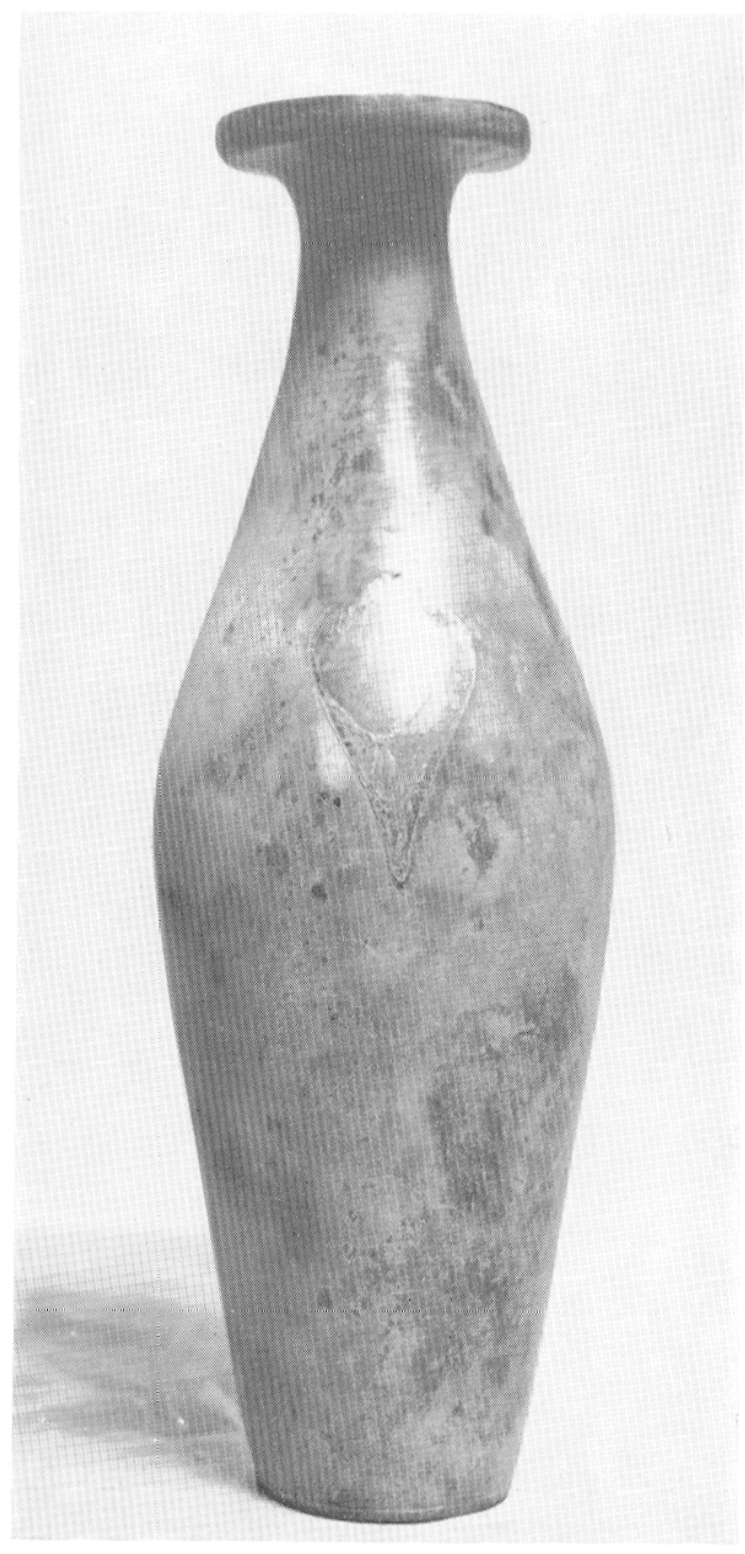

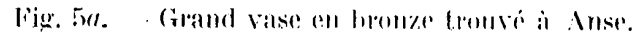

18:24 el exposé au Musée Denon's. Les deux récipients onl presque la mème hauleur (0,33 a Anse el 0,31 à Chalon), le mème galbe el ont pareillement perdu leur anse: dont l'arrachement a laissé en bas une empreinte cordiforme. Ils ont les mèmes cercles concentriques fondus en relief sous le lond. Peut-ôtre les deux ustensiles sortent-ils du mème atelier puisqu'ils ont non seulement le mime profil mais les mêmes délauls de labrication. La patine dans les deux cas est verte, plus belle ì Inse qu'à Chalon oil de gros grains de sable ont adhéré fortement partoul. Ces vases sont des anochoés it anse de lorme probablement, anguleuse, ce qui incilerait à les dater du milieu du II $^{\mathrm{e}}$ siècle ${ }^{14}$.

3. ... Une patere en bronze (lig. 5), a g.), haute de $0,0: 57$, longue de $0,29 \%$ avec la queue, a élé décorée, vers l'extrémité de relle-ci, de ciselures au trait, representant des godrons, des hachures el des fleurelles. 'Trois rercles concentriques en creux sont visibles sous le fond. Aucune patine ne s'est formée sur le mélal qui paraîl avoir élé doré et auquel adherent seulement quelques dépoits noiràtres. Nous

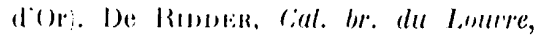
$11^{\circ} 2765$ andrien. Rhòne?.

13i lal. du Musée de Chalon, onv'. cite. $\mathrm{n}^{\circ} 435$.

It Voir la stide publice dans la lime. arrhenlengique. 1937. :- p. 22:3 et le vase 11" f0: du Musie de chalon ainsi que linnochoe du louvre fde limbl:k, rial., II, n $^{\circ} 2697$ et pl. 98), trouvese "remplie de monnaies du temps dlladion". 


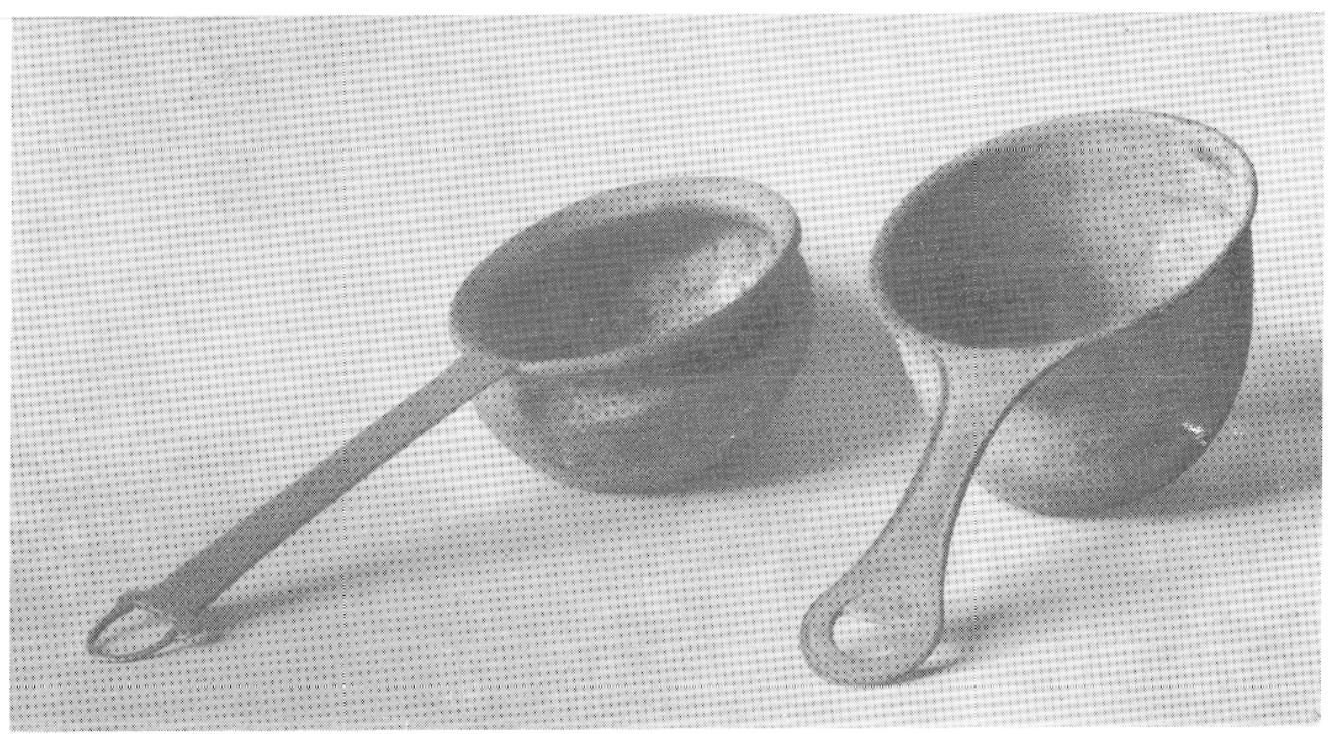

lig. 5b. - Patire el casserole d'époque romaine tronvees à Anse.

pensons pouvoir dater cet ustensile du

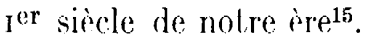

4. -.. Une casserole (fig. $\overline{5}^{\mathrm{L}}$, a dr.) mesure 0,247 avec la queue. Sa hauteur est de 0,077. Cette pièce de vaisselle est pourvue de cing cercles conceniriques en relief sous le fond et de deux autres plus pelits, el creux à l'inlérieur. Belle patine verte. L'exlrémité de la queue est ajourée d'un demi-cercle qui monlre des traces d'usure altestant que cette rasserole a élé suspendue à un clou ou bien à un crochet. La forme générale de l'ustensile (el singulierement en ce qui concerne la queue dont les bords latéraux sont relevés) appelle la comparaison avec le $\mathrm{n}^{0} 16$ de Radnoli qui date ce modèle de 50 à 150 après J.-C. ${ }^{16}$.

(15) A. Radnot, lie römischen Bronzegefässe von Pannonien, Budapest, 1938, pl. 2, $n^{\circ} 7$.

(16) Ibid., pl. 4. On notera que le catalogne par líönner de la coll. J. (iréau, Bronzes antiques, 1885, p. 11, $1^{\circ} 35$, fig., mentionne une casserole en bronze trouvé a Anse, qui s'ajoute ainsi à celles ici décrites.
5. - Une hache en fer (fig. 6, id g.) de charron ou de bûcheron, longue de 0,175 , peul ctre attribuée à l'époque romaine, car elle est semblable à celle du Musée des Antiquités nationales (no 28.991c) trouvéce dans la forêt de Compisigne ex à une aulse que nous

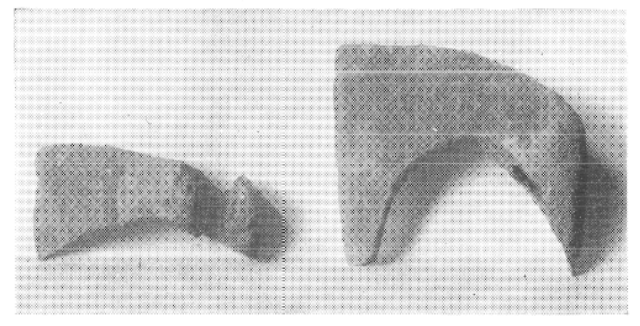

Fig. 6. Iaches en fer gallo-romaines trouvées à Anse.

avons recueillie dans les fouilles de Marloux, associéc à du gallo-romain précore el à du gaulois ${ }^{17}$.

(17) S. Renacin, Cal. du Musee des Anl nat, 20 éd., I, fig. 272, n०28.991", et Gallia, 1944, p. 33. 
6. -.. Une autre hache de fer (fig. 6, a dr.), longue de 0,18 , rappelle certains outils romains " de bùcheron ou de sabolier" de la mème collection $\left(\mathrm{n}^{0} \quad\right.$ 1940) ainsi qu'une hache découverte an sancluaire de Sanxay (Vienne) ${ }^{18}$. Celle forme, caractérisse par une forte courbure inférieure el une hauteur fort grande du tranchant (ici 0,15$)$, est rare.

7. Uh vase en lerre noire, sans anses, haul de 0.12 el de profil caréní, apparlient à un lype de céramique die tradilion reltique que M. J.-.J. Mall date de Claule is Domitien' ${ }^{19}$. (fiz.. 7, à g. . poleries régionales. I.es deux exemplaires, ici reproduits, el dragués à Anse, semblent de bassé épocque (rve siècle?) ${ }^{20}$; d'aulres pourraient être du ve.

Eporque barbare. Aux temps harbares se ratlachent:

1. - Vne grande pointe de lance (fig. 8), longue de 0,40, mérovingienne ou carolingienne.

2. .... In vase en forme d'onochoé, a bee trellé (fig. 8, en bas) ea terre rougeailre et orné a la rouletle de dessins géométriques que surmontent des trails
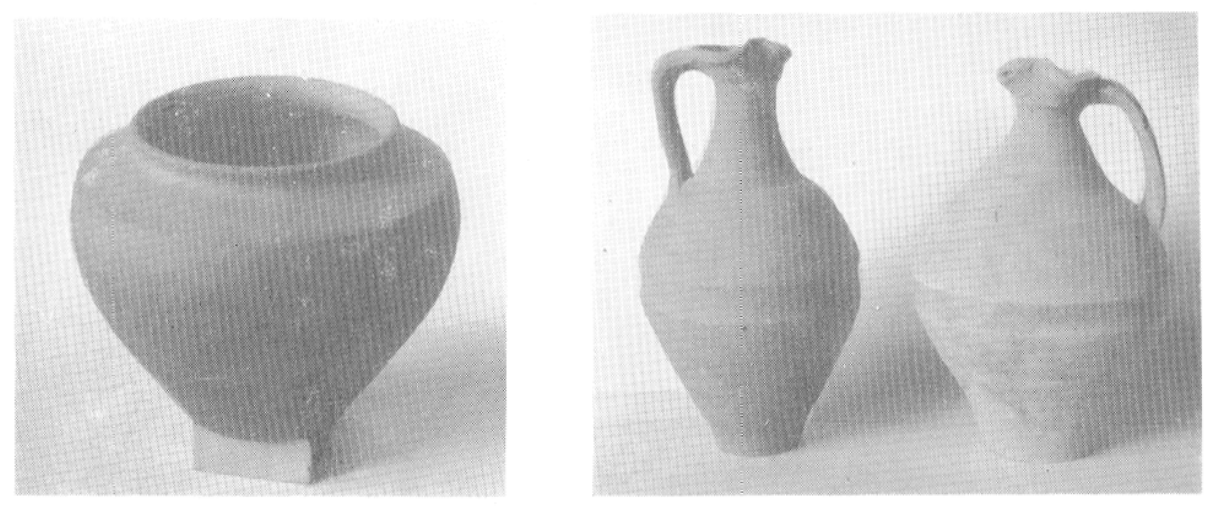

Fig. 7. - Vases en terre cuite trouvés à Anse.

8. Plusieurs anochoés en lerre rouge it bec trèllé (lig. 7 , a dr.), hautes de 0,29 , ont comme caractéristique essentielle une cannelure horizontale creuse vers le milieu de la panse. Ce type, qui paraît rare ailleurs, est très fréquemment rencontré dans le lit, de la saòne. entre Chalon et Lyon. Le Musée de Chalon en posside d'assez nombreux spécimens qui doivent provenir de

(18) Rl:sхucu, Cal. cile, fior. 272, no 19405, et Eycion, dans Gallia, II, 1944, p. 102, fig. 7, no 12 .

(19) J.-J. HАтт, Rev. Et. anc., I.I, 1949, pl. X, nos 23 et 24 . horimontaux maladroitement tracés. II. $0,21^{21}$.

Ces decouverles fortuiles paraissent

20) Vime an!. pl. XII, $n^{\text {os }} 23$ et 24 . (i) Bull. des Anl. de firance, 1933, pl. 133. Cette gorge horizontale a cependant des orjgines lointaines qui remontent a la periode flavienne (79) a 97 , apris .J.-(:), voir aussi llatr dams Ciallia, XII, 195.1, 2." part., p. 328, fig. 7, $1^{\circ} 4$.

21 Lin achevant cet inventaire des trouvailles faites par M. Rebut, nous devons signaler que V. Ie Maire d'Anse nous a montré dans son petit. musere municipal une epé de hronze incomplets et plusieurs poinles de lance en fer, paraisant. les unes ganlorises, les antres mérovingiennes. les armes, qui proviennent de la Saone, anmient. diti dagueses dans lew mômes parages. 


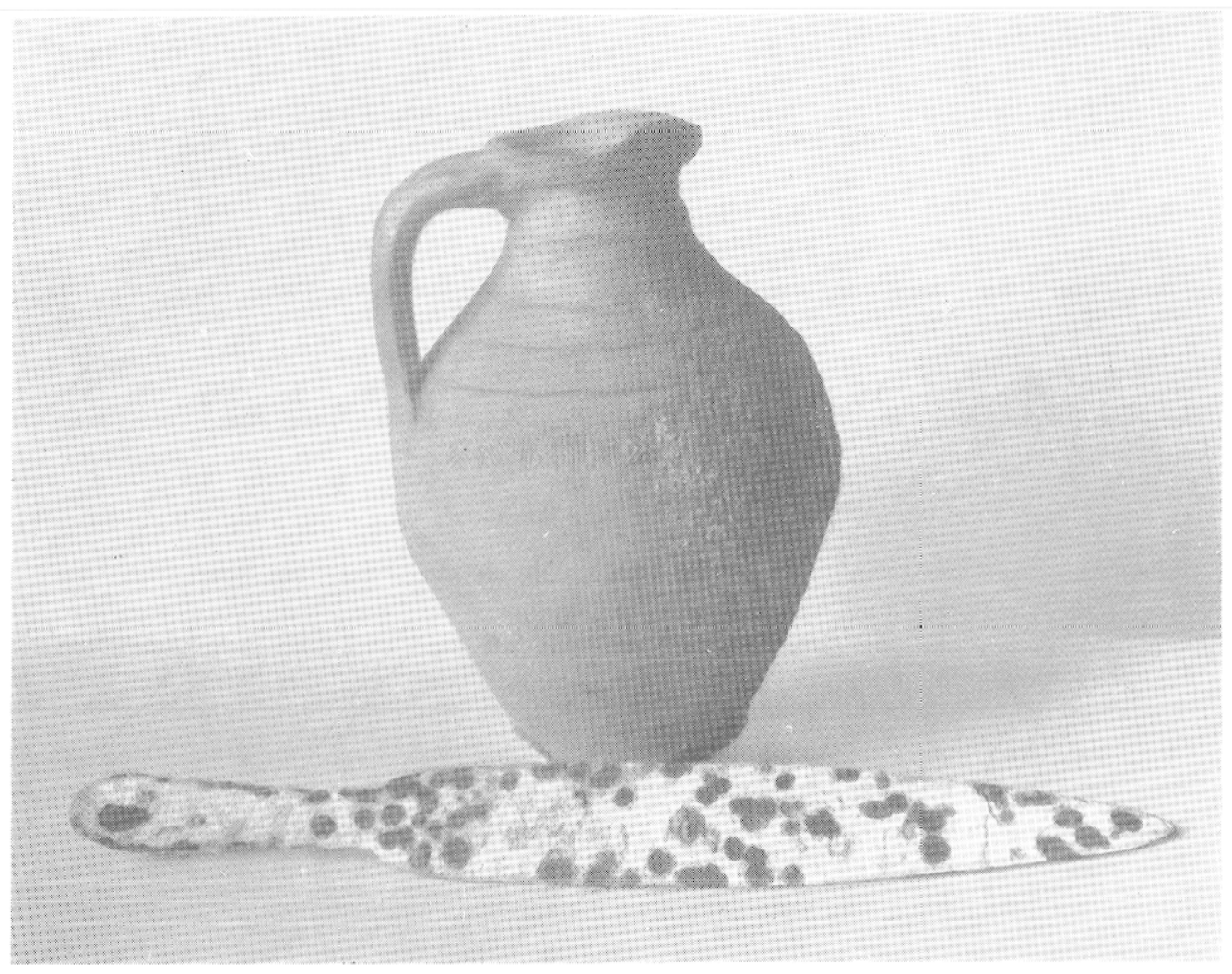

Jigr. 8. Pointe de lance et poterie mérovingienne lrouvies à Anse.

révéler l'existence, légèrement en amont. d'Anse, d'un lieu de passage sur la saòne, en relation, dis les lemps probohistoriques, avec les élablissements hallstatliens de Saint-Bernard, et plus Iréquenté encore pendant l'occupalion romaine. Mentionné en cflet par l'itinéraire d'Anlonin. le caslellum d'Anse, sur la grande route de Lyon a Boulogne, était le point d'aboutissement d'un chemin secondaire qui, grefle sur la voie d'Agrippa, reliait la Saòne à la Loire par Légny, Saint-Clément, Amplepuis, Pradines et lioanne22. Les riches mosaï-

(22) Pierre Fustuk, Rev, arch. de l'Est, 1955, p. 71. Voir surtout (.. Juindian, Les problemes d'Anse, dans la Rev. des Et. anciennes,

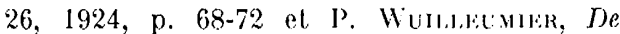
Llyon à Mâcon, ibid., 41, 1939, p. 245-251. ques mises au jour sur son territoire ${ }^{23}$ sont parlois ornées d'altribuls nautiques ou aquatiques (ancres, proues de navire, dauphins) qui pourraient rappeler le caractère particulier de l'activilé régionale, de mème que cerlain plomb trouvé dans la saòne, qui désigne vraisemblablement les Asenses, c'esta-dire les habilanls d'Asa Paulini, sous la forme Ansenses, montre l'importance. religieuse el commerciale d'Anse's4. Si

(23) A. BLANchis, Ine. des mosaïques de In Gaule. II, 1909, p. 16-17, nos 766-767.

(21) A. Grenilir, Manuel, Il, 1934, p. 657, nole 1. Cr., même ouvr., J, p. 445 et 457. - - Le plomb porte $A N S L N S$, ce qui donnerait Ansenses, alors que l'Itineraire d'Antonin porte Asa et non Ansa; mais M. BI.ANCHIT (Bull. des Anliquaires de France, 1904, p. 150) remarque que l'Itincraire fournit de mauvaises lecons. 
l'on songe en outre que Jullian voyait dans le nom $A s a$ un mol signifiant "quelque chose comme porl ", il apparaît. possible non seulement qu'un port, mais que plusieurs ports ${ }^{25}$ aient existé autour d'Anse et qu'ils aient été ulilisés jusqu'à la période mérovingienne. Cependant le lieu où ont élé dragués les objets considérés ne saurait être le port principal que Jullian silue avec vraisemblance a l'embouchure de l'Azergues, c'est-ii-dire plus près du bourg el dans une position mieux lavorable au mouillage. Comme l'endroil, faisant face à La Bruyere el dont les Irouvailles ici décriles ont montré l'inlérèt, se place dans l'axe d'un chemin venant de la vallée du Formans el se dirigeant vers Bourdelan d'Anse, il semble probable que c'était le point où ce chemin (d'origine préhistorique, mais sans doute encore suivi pendant l'occupa-

Jullian (article cilé) croyait que ce plomb est un jeton d'entrée à des jeux, ce qui prouverait fu'Anse "retait, comme Alesia et d'autres bourgades, un rendez-vous de spectacles of partant, un sanctuaire et un lieu de marchén.

(25) Nous avons constati qu'il en itait ainsi aux abords de chalon et que ces ports se confondent souvent arec des lieux de passage, notamment avec des gues (roir L. ArmavnCaldiat, Ia Saône el l'hisloire, dans les innalos de Bouryogne, 1951, p. 111). Sur le deplacement des ports, voir Jituras, VIII, p. 2:26. lion romaine) franchissait la Saône au moyen de baleaux ou d'un bac : rien ne subsiste des pilotis d'un pont et c'est seulement à 2.200 mètres en amont, vers Riotier, que se trouve. paraît-il, un gué.

\section{GERTiY (SAÒNE-ET-LOIRE)}

M. Namin, garde champètre de Gergy, a fail don tout récemment au Musće de Chalon d'une série d'ohjets de l'àge du Bronze, de IIallstatt et de La Tène qu'il a recueillis en 1955 des mains des dragueurs opérant sur la saòne à la hauteur du territoire de celte commune siluée à une douzaine de kilomètres en amont de Chalon. Il nous a fait connaître également quelques pièces gallo-romaines et barbares de même provenance. Voici l'inventaire de ces trouvailles.

Bronze el Ilallstall. -.-. 1. ..- Petite épée en bronze i soie plate avec rebords relevés (fig. 9). I. 0,41. I)ans son état. acluel, la poignée ne comporte que quatre trous ayant, le plus souvent la forme d'une fente. Seul l'un d'eux, le plus petit, a conservé son rivel. La lame est. pistilliforme avec échancrure a sa base ; la patine, vert olive. Bronze IV de Décheletle.

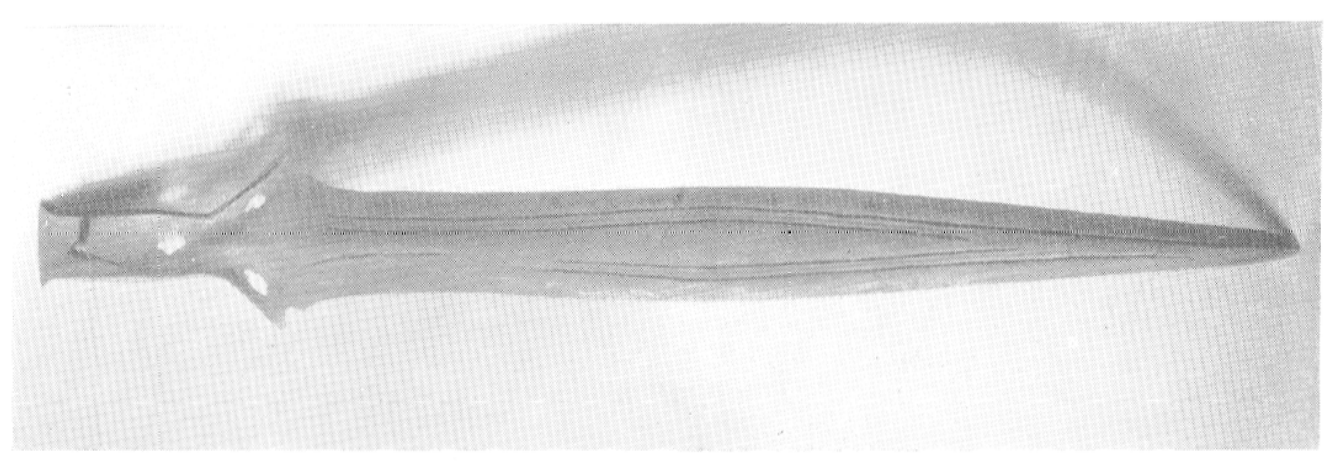

Fig. 9. - - lipé de l'âge du Bronze trouvée au gue de Bougerot pris de Gergy. Musée de Chalon. 
2. Vase en lerre noire, haul de 0,064 (fig. 10, à g.) fail d'une pâte asse\% grossière, mélangée de grains de quarl\%, et modelćc à la main. C'est une tasse dont la queue a élé brisée, laissant un double arrachement très nel. La civilisalion des Champs d'urnes a produil souvent des petits récipients de ce
Il n'est pas sans évoquer non plus le matériel hallstallien de Las Fados (Aude) $)^{28}$.

La Tène.-1.- Épée de fer (fig. 11, en bas), longue de 0,865. La croisière est. campaniforme el la soie terminéc par un boulon. L'extrémité de la lame

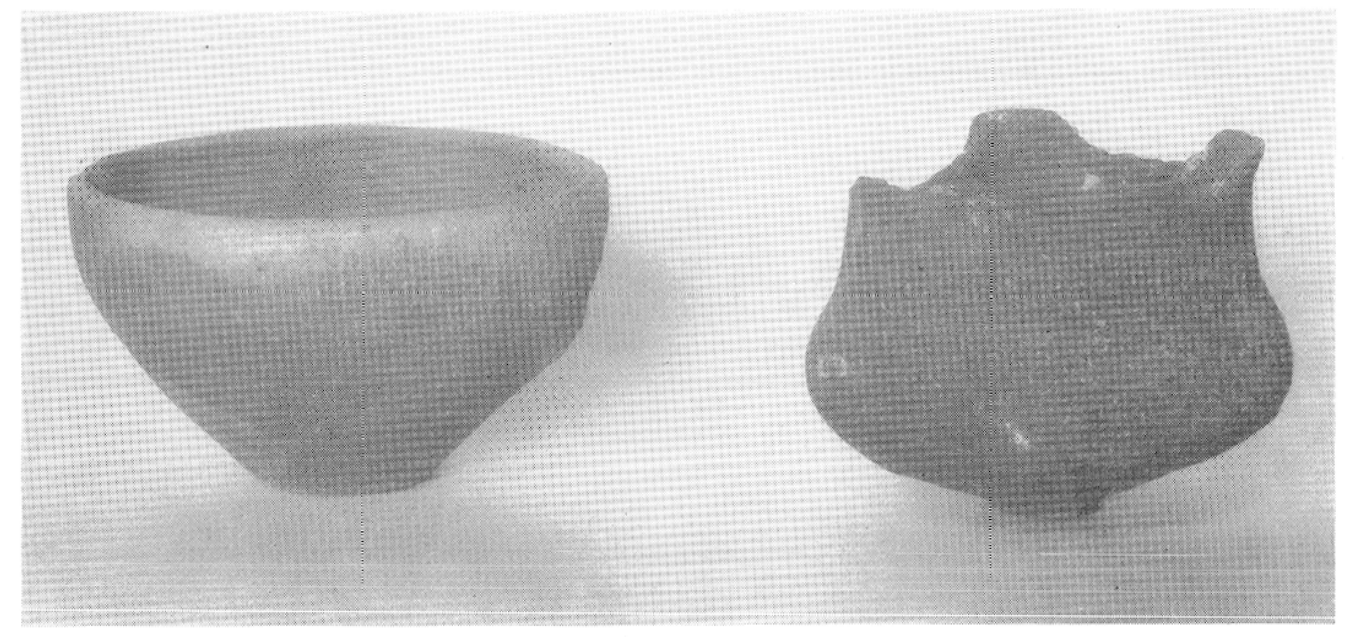

Fig. 10. ...- Vases de l'époque des champs d'urnes trouves au gué de Bougerot pris de Gergy. Musée de Chalon.

genre ${ }^{26}$, dans la Marne el l'Oise nolamment.

3. - Aulre vase en lerre noire, obtenu également sans l'aide du tour el conservant dans sa pâte de gros grains de quartz (fig. 10, à dr.). II. 0,007. Il est. sans anses, de profil caréné, a pied très étroit, creusé par-dessous. Plus original que le précédent, il s'apparente pourtant avec certaines poleries provenant des incinérations sous lumulus de Bouzemonl. (Vosges), que M. Kimmig date de la période tardive des Champs d'urnes" ${ }^{27}$.

(26) Krmma, Rev. arch. de l'ksl, 1952, p. 146 (Betheville); 1953, p. 210-211 (Aulnay); 1954, p. 225 (Saint-1'ierre-en-Chastres). Cette dernière station est l'cpoque des Champs d'Crnes II-III.

(27) Ibidem, 1952, p. 161, figr. 31, $1^{\circ} 7$ (fin de Ifallstat ). n'est pas poinlue ni tout i fait arrondie. mais bien triangulaire. I,a Tène II.

2. Grande poinle de lance, draguée avec l'épée précédente el la pointe de lance suivante. Longue de 0,54 , elle est caractérisée par sa forle nervure médiane tranchante (fig. 11, au milieu).

3. --. Seconde pointe de lance (fig. 11, en haut) de même origine et de mème lype mais plus pelile (L. 0,47$)$. La nervure médiane est moins accentuéc.

4. - Parlie supéricure d'une anphore (fig. 12) en terre grisàtre, recouverte d'un engobe blanc. Maut. 0,13. I.es

(28) O. et J. Tafraner, Gallia, VI, l918, p. 16, fig. $18, n^{0} 50$. 

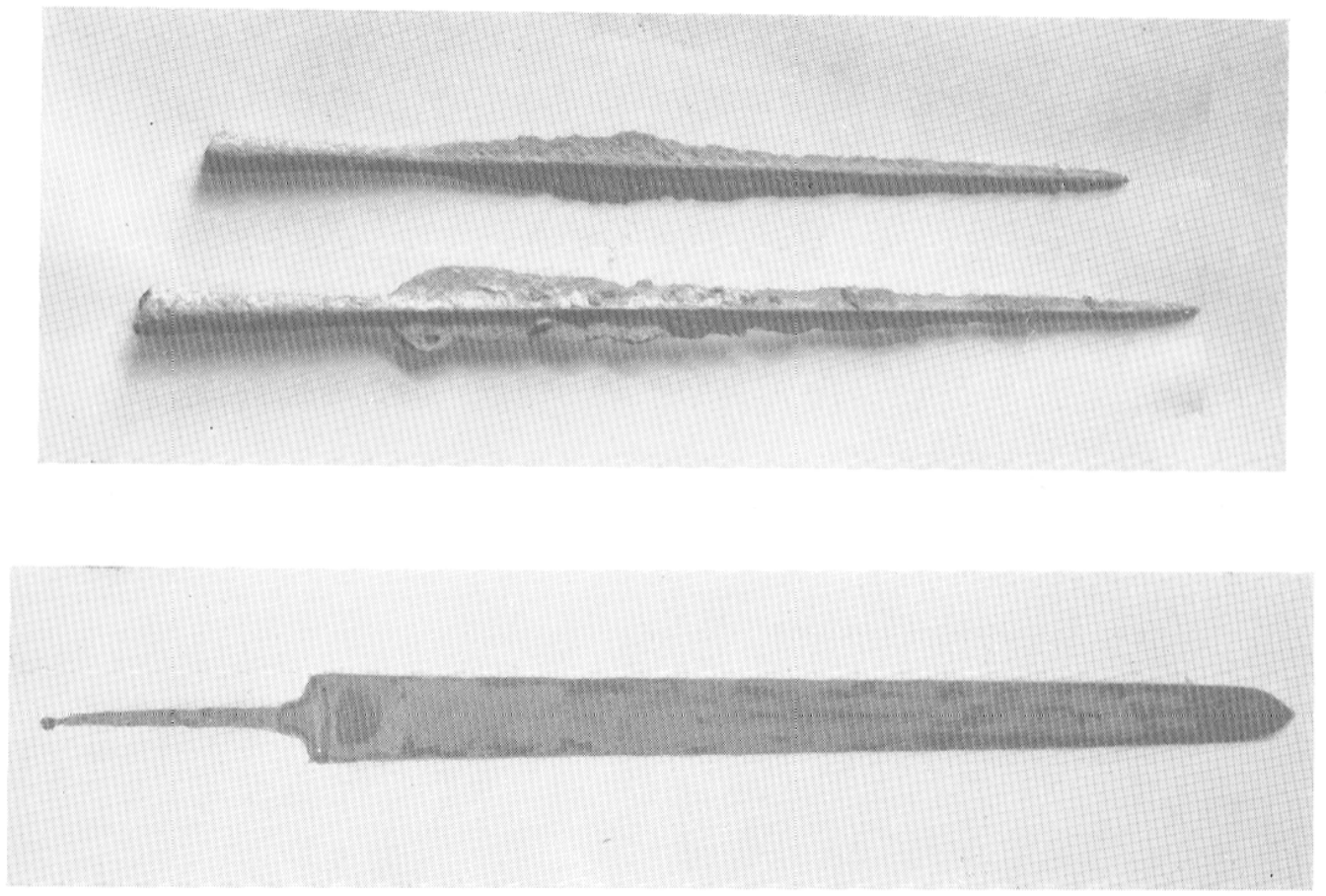

Figy. 11. Pointes de lance gauloises et épée de La T'ene II, recueillies an gué de Bougerol. Musée de Chalon.

anses plates, minces et droiles, sont triparliles. Le goulot, très rétréci, lui donne un profil fort curieux dont nous ne connaissons guère d'exemple. L'aspect. des cannelures triples sur les anses el surtoul la qualilé de l'engobe, très pulvérulent, nous font attribuer ce débris ¿ La Tène III ou au gallo-romain précoce $^{29}$.

Epoque yallo-romaine. . 1. . Fond de vase en terre sigillée portant la signalure OF. MACGA. Probablement s'agit-il de MACGARVS, polier qui, à la Ciraufesenque, Iravailla de Tibere ¿ Véron.

2. Poleries communes diverses que nous avons vues, mais qui ne sont. pas entrées non plus au Musée Denon.

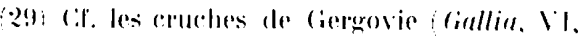

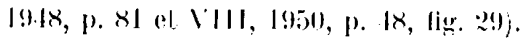

Éporque barbare. Cinq objels de ler, incontestablement mérovingiens et restés aussi a Ciergy, paraissent constituer

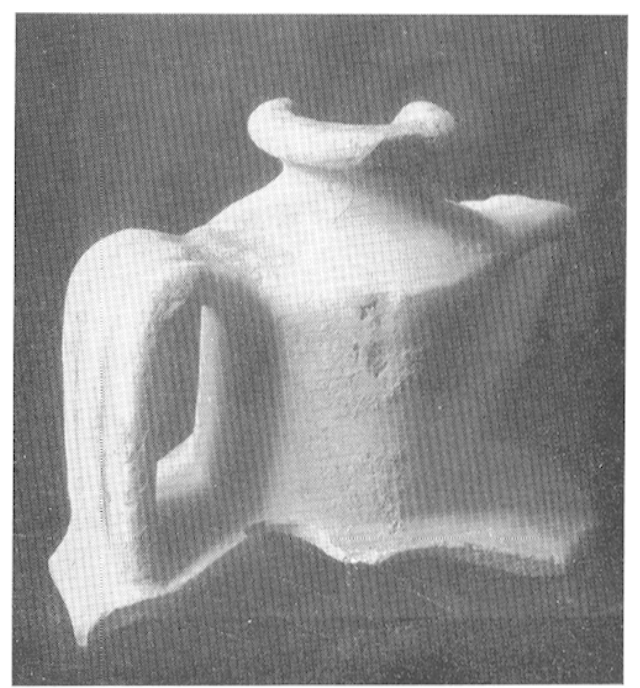

Fig. 1:. (:0) d'amphore (1.a Tene III ?) fromai iul gue de Bougerot (1[r 0,13$)$. 
l'équipement d'au moins deux guerriers qui onl dû périr dans le lleuve, car deux crânes humains ont été ramenés en même lemps par la drague.

1. - Epieu ì crochets. L. 0.43.

2. Nutre pointe de lance sans (rochels. I. 0,2).

3. Scramasax ¿ tranchant droit et dos rourbe. L. 0.41.

4. -.. Coul eau Iris usé. L. 0,22.

¿. Aulre couteau plus pelil. I. 0, 15.

Tous res objels protohistoriques, galloromains el mérovingiens onl élé dragués dans la sacone en anout 19\%5, sur un

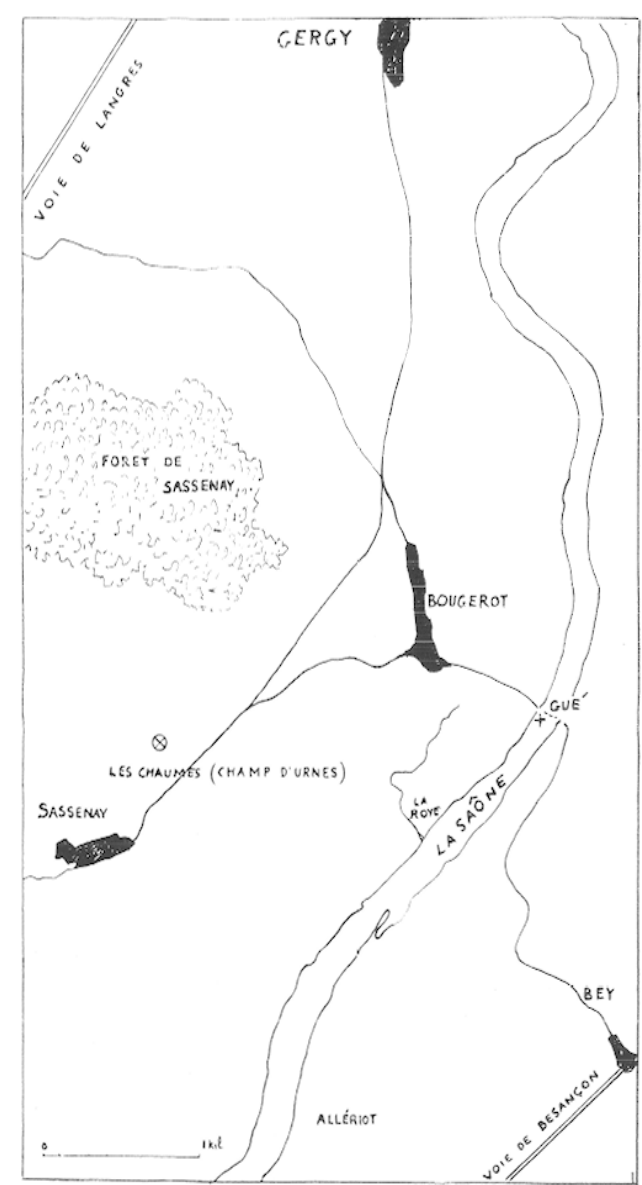

Fig. 13. - Carte de la région de cergy indiquant l'emplacement des trouvailles all gué de Bomgerot. espace tris limité (fig. 13), se plaçant. a I kilomitre au nord de l'embouchure du ruisseau nommé la roye de Bougerot, sur la commune de Gergy, c'est-a-dire approximativement à la hauteur de l'enceinte néolithique ou chalcolithique de Bougerot ${ }^{30}$, formée d'un rempart en terre dessinant une ellipse appuyce au rivage. L'accumulation de ce matériel archéologique, et en parliculier celui des armes, dans le lit de la riviere s'explique par l'existence d'un gué que les ouvriers de la drague onl repéré au mème licu el qui a été certainemenl. le théàlre de combals a l'époque barbare. après l'avoir élé dès l'âge de Bronze el, l'àge du Fer ; car une coulume observée. chez les Celtes comme chez les (iermains voulait que les engagements militaires et les combals singuliers se livrassent. de prélérence non seulement. sur less bords, mais au milieu même des eaux ${ }^{31}$. la lulte pour les gués est le type par excellence du combat celtique, et l'histoire nous apprend que les passages de la Saone élaient l'enjeu des guerres entre les Éduens et les Séquanes ${ }^{32}$. Les chroniqueurs du xrre siècle se fonl encore l'écho de la persistance de cel usage mililaire, commun aux Gaulois et aux (iermains el qui se perpétua pendant le Moven $A$ ge proprement dit. Dans les épopées irlandaises, le duel ou le combat sur

(30) Diclionnaire arch. de la Ganle, p. 44. M. le 1$)^{r}$.1. (arlot. de Verdun, a reconnu dernièrement celte enceinle, et nous lui devons d'autres précisions notamment au sujet du gué, les drageurs de l'entreprise llontupet, dont le sioge est à Verdun, lui ayant fait des déclarations formelles i ce sujet.

(31) Rene locis, line coulume dorigine prehishorique, les combals sur les guis chez les Celles el chez les Crermains, dams la Rev. arrh.

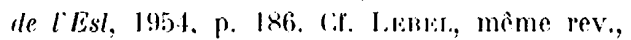
1953, p. $35 \%$

(32) Strabon, IV, III, 2. (:I. Jitilax, III, p. 138 , ก. 6 . 
le gué possède un caractire riluel, c'esl une sorte de "jugement de J)ieu", une ordalie par laquelle la divinite lluviale decide entre les adversaires; aussi n'aurait-on pas osé reprendre au cours d'eau ou au génie qui le personnifiait les armes lombées et devenues en quelque sorle labou ${ }^{33}$. Ces textes permetlent de comprendere la lrouvaille d'un rasque du vi"siede ol d'une lance ou d'un angon sur le gue de la Truchere au voisinage de 'lournus ${ }^{34}$, al celle d'armes merovingiennes diverses all gue d'Allériob ${ }^{3 i}$. Les déconvertes récentes de Bougerol que nous venons d'énumérer

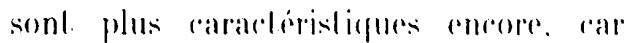
elles mellent en lumiere la prénenile d'un passage de la riviere pendanl

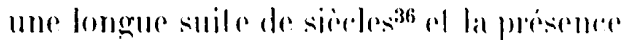

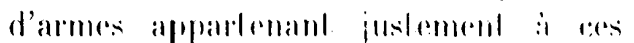

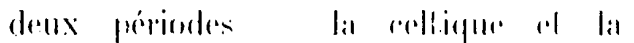

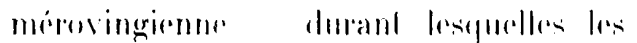

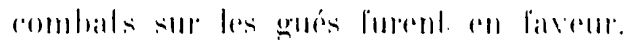
Au resler, and il ne laul jamals systoma-

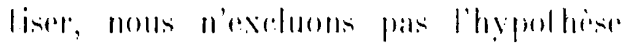

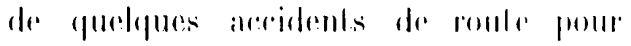
une partie an moins des tromvalles d'objels divers, ni celle de l'existemere de quelgues postes de prage? rele relle olservalion est valahle allssi bien a Anse qu'a Bongerol. On nolera relleore que prese de Rarenmaly thameane de firergy rommer Bongerol al rommme lui

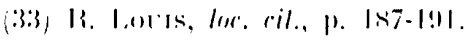

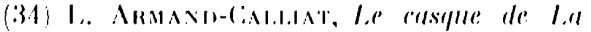

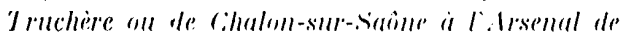
Berlin, dans le Bull. de la Sociele des Amis des Arls de Tomrnus, 19.11, p. 193. Nous avioms des lors emis l'hypothese que ce castule? avait dì Âle perdu au cours d'un rombat en 5ot.

(35) J.e Chalommais gallo-romain, 19:37, p. 85.

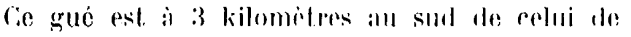
รlomererot.

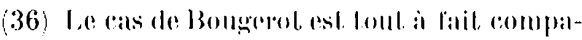
rable a celui de Try, pres de loormans, sur la Marne. (.f. R. Gavintrox, Bull. de In Sociéle des Anliquaires de Fromre. 194:3-19.14, p. 395. silué au bord de la Saòne) se trouve le lieu-dil, La Varande, loponyme vraisemblablement dérivé d'Éyuaranda, mot. prélalin auquel on donne le sens de "limile d'eau "37.

Il n'est pas sans intérêt non plus de jeter un coup d'reil sur les trouvailles failes précédemment autour de Bougerot, ef du gué qui nous occupe. (In a déjii recurilli la beaucoup de haches polies (donl lrois sont au Musee de Chalon), des poinles de fleche néolithigues en silex linement laillé (exposées dans les mèmes colleclions), ainsi qu'une épée de bronze draguée dans la saine on 187:38. l'n lerber de sable, appele lo lumulus de Bongerol, a livé des monnaies de Vespasien, de Mare-Auriele. de Commode ol. de Comsanlin. ollerles all Musere lemon par la habilanl du pays. V. Salequerl. Vous avons remarqué allsid.

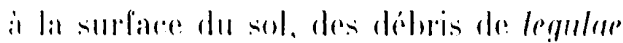
al de rondeils d'hyporansle ; rnlin on a dilerre, avanl lefo, un sarcophagre de eries el des plapues-bondeles damalspuineses mérovingiennes39. II ronvienl de rappeler d'aule pare, ele leail cost. imporlanl, que le champ d'urnes de

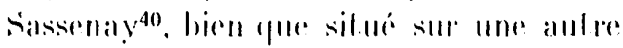

37: Voms lavous sigmale dans les Mem.

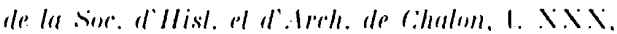

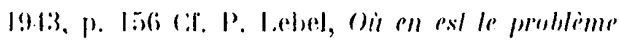

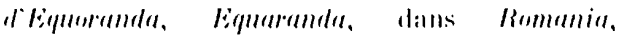
I. I. X111, 19:37, 1. 115.

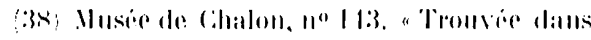

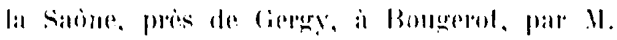
Iommarlin, de Verjux".

39! l.e Chalommais gallo-romain, p. 158.

(40) Voir, outre Ciallia, I, 1943, p. 31-32, notre note daus la liev. arrhéol. ale l'Esl, 1952, p. $96 \mathrm{ct}$ Kinure, même recueil, même année, p. 144. La figr. 24 ile ce dernier article contient une double erreur : le troneon d'épée provient de Granges ef le couteau de Sassenay. D'autre part, nous avous retrouve l'emplacement précis du champ d'urnes de Sassenay sigmale par Fre Cinabas, dans un manuscrit inedit: c'est le lien-dit Ios Chanmes. 


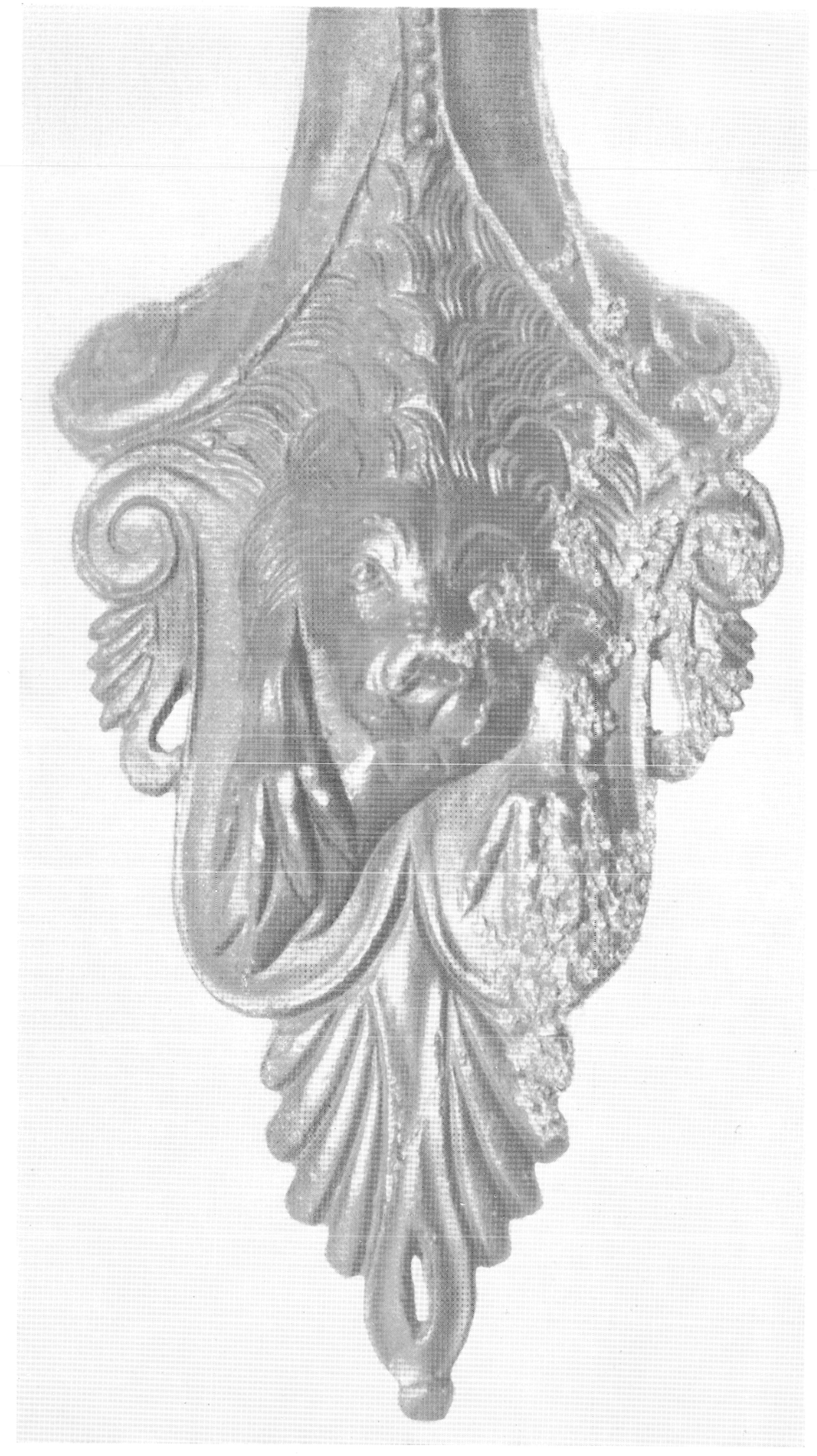

Fig. 14. - Anse d'œnochoé trouvée dans la Saône près de Chalon. Musée de Chalon-sur-Saône. Hauteur : 0 m. 27 , 
commune, n'est pas éloigné de plus de $9 \mathrm{~km}$. 500 el se place sur la rive droile également.

En somme, a Bougerol comme a Anse, on est en présence de dépòts archéologiques dans la saòne qui sonl, certainement en relation avec les établissements prolohistoriques ou hisloriques riverains. Ces gisements lluviaux ont pour origine des bac's (. Inse) ou des gués (Bougerol) lréquenles pendant les momes périodes que les habilals loul. proches. Fi rese relle concordaner qu'il serail bien inléressanl d'éludier loul le long du cours de la saone, si les déconvertes eflectueses par less dragueurs nous elaient, chacpue lois signalées.

\section{SUD de: GHalon}

Pour lerminer, nous décrirons ici une anse d'enochoé en bronze (fig. 14) donnée, comme les anliquités protohistoricues de Bougerol, au Musée de Chalon par M. Namin. Lille a élé draguée, il y a quelques années déji, au sud de. Chalon. Son principal mérite réside dans ses dimensions, lries supérieures à la normale : 0,27, surlout pour une piece ciselée. litle est ornée, à la base, d'une protomé de lion et de palmettes; en haut, d'un reptile enroulé, donl la lèle manque. Le fauve dévore la palte d'un animal qui pourrail ètre un sanglier. Ce molif est à comparer avec le "manche de clel ou de miroir", lrouvé à Berthelming (Moselle) parmi les ruines d'une villa el, representant "un chien qui lient dans sa gueule un pelit

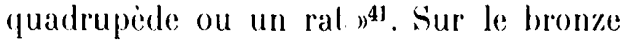
lorrain, le pelage a été trailé en effel avec une lechnique lres analogue is celle qu'on remarque sur le bronze chalonnais. Quant au thème du lion dévorant, une proie animale, theme auquel on accorde volonliers en Gaule un caractère in lernal ${ }^{42}$, loul comme it relui da monstre androphage, il ne parail pas avoir souvent, conslilue ches nous un ornement d'anse de vase ${ }^{43}$. l'aul re parl, la forme générale at la dimension de l'anse monlrent que le vase complel devail mesurer plus de 0,11). ree qui dépasse de bealucoup la movenne

Iu lolal, ces documenls archéologiques, relirés des sables sur loul le cours de la rivière, peuvent lournir une contribution à l'étude de la protohistoire et de l'histoire saoniennes. Puisse leur publicalion inciter a une surveillance plus étroite des découvertes!

\section{Louis drmand-CialdidT}

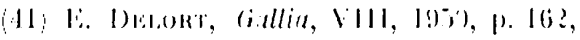
fị... 5.

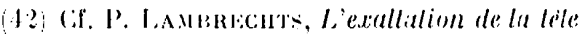
datus la pensege el dans l'art des Celles, Bruges, 1954, p. 62 et 11 . Rexard, finues androphnges, dans Ilommages à .J. Bider el à $F$. Citummnl, colll. L.alomus, II, p. 282.

(43) comme ornement de coutenu, voir

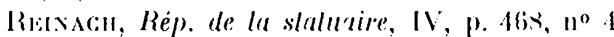
(Gence) 474, $n^{\circ} 4$ (Arovie, $177, n^{\circ} 1$ l le Caire: d'ustensile, V, p. 427; de vase (mais non d'anse. IV, 478 (Venise).

(44) Voir au Vusede de (hallon, une anse unie trouvée dans la saone $\left(1^{\circ} .111\right)$ qui mesure 0 m. 2.1 mais ne présente aucune cisclure. 